\title{
Assessment of Panoramic Radiomorphometric Indices of the Mandible in a Brazilian Population
}

\author{
Maria Beatriz C. C. Alonso, ${ }^{1}$ Arthur R. G. Cortes, ${ }^{2}$ Angela J. Camargo, ${ }^{2}$ Emiko S. Arita, ${ }^{2}$ \\ Francisco Haiter-Neto, ${ }^{1}$ and Plauto Christopher A. Watanabe ${ }^{3}$ \\ ${ }^{1}$ Department of Oral Dignosis and Dental Radiology, Piracicaba Dental School, State University of Campinas, \\ 13414-018 Piracicaba, SP, Brazil \\ ${ }^{2}$ Department of Oral Radiology, School of Dentistry, University of São Paulo, 05508-9000 São Paulo, SP, Brazil \\ ${ }^{3}$ Department of Morphology, Stomatology and Physiology, Ribeirão Preto School of Dentistry, University of São Paulo, \\ 14040-900 Ribeirão Preto, SP, Brazil
}

Correspondence should be addressed to Plauto Christopher A. Watanabe, watanabe@forp.usp.br

Received 16 May 2011; Accepted 4 July 2011

Academic Editors: A. Adebajo and E. Tchetina

Copyright () 2011 Maria Beatriz C. C. Alonso et al. This is an open access article distributed under the Creative Commons Attribution License, which permits unrestricted use, distribution, and reproduction in any medium, provided the original work is properly cited.

\begin{abstract}
The aim of this study was to evaluate radiomorphometric indices in dental panoramic radiographs in order to identify possible interrelationships between these indices and the sex and age of the patients analyzed. The study included 1287 digital panoramic radiographic images of patients that were grouped into five age groups $(1=$ age $17-20 ; 2=$ age $21-35 ; 3=$ age $36-55 ; 4=$ age $56-69$; $5=$ over age 70). Two indices — cortical width at the gonion (GI) and below the mental foramen (MI)—were measured bilaterally in all panoramic radiographs. Statistical analysis was performed with Kruskal-Wallis and Mann-Whitney tests (alpha $=0.05)$. Results for the indices measurements showed significant differences among patient age groups of both sexes, considering that groups 4 and 5 presented lower values for the cortical width of both indices. The present paper supports the role of sex- and age-related changes in mandibular radiomorphometric indices in identifying skeletal osteopenia.
\end{abstract}

\section{Introduction}

Osteoporosis is a well-known and common systemic skeletal disease, characterized by low bone mass and microarchitectural deterioration of bone tissue, with subsequent increase in bone fragility and risk of fractures [1]. Bone tissue undergoes constant change caused by the simultaneous processes of decay (resorption) and formation (regeneration), generally termed as bone remodeling [2]. This turnover ensures the continuous replacement of old bone tissue, which, in turn, affects bone adaptation to various mechanical forces exerted on the skeleton [3].

The detection of an eroded or thin inferior cortex of the mandible on dental panoramic radiographs may be useful for identifying patients with low bone mineral density (BMD), skeletal osteopenia, or osteoporosis [4, 5]. A number of studies have revealed significant correlations between $\mathrm{BMD}$ of the mandible and that of the most frequent sites of osteoporosis, namely, the lumbar spine, the femoral neck, and the forearm $[1,4,6]$.

Although osteoporosis has been established as an important disease in postmenopausal women $[7,8]$, its effect on mandibular bone quality has also been described by a few studies in men $[9,10]$, inferring that sex and age are factors that may influence BMD and the prognosis of osteoporosis $[11,12]$. Nevertheless, details on the difference between results for male and female patients of different age groups remain unclear in the literature.

BMD may be assessed using modern recognized techniques such as dual X-ray energy absorptiometry (DXA) $[13,14]$. However, osteoporosis may also be diagnosed by analyzing radiomorphometric indices on a panoramic radiograph-an accessible image-obtaining method frequently used in general dentistry. These indices are correlated with mandibular BMD $[7,8,14]$. 
Thus, the present study aimed at evaluating the measurements of panoramic radiomorphometric indices of the mandible in different age groups and also at comparing the results for male and female patients.

\section{Materials and Methods}

2.1. Sample. The patient sample was selected from the archives of the Ribeirão Preto School of Dentistry of the University of São Paulo and consisted of males who had undergone a dental panoramic radiographic examination between March 2007 and June 2010 as part of their dental treatment. Records and digital dental panoramic radiographs (DDPRs) of 1287 patients were examined. Of these patients, 503 were male, and 784 were female. Patients were previously grouped according to five age groups $(1=$ age $17-20$ years; $2=$ age $21-35 ; 3=$ age $36-55 ; 4=$ age $56-69 ; 5=$ over age 70 ). All DDPRs had been taken using a Veraviewepocs Digital panoramic radiography unit (J. Morita Co. Digital, Brazil). This study was approved by the Ethics Committee (CEP) of the Piracicaba Dental School, State University of Campinas.

2.2. Radiographic Assessment. All panoramic radiographic measurements were performed by a single trained observer, using a specific computer software program (Radioimp 2.0, Radiomemory, Belo-Horizonte, Brazil). The measurements were compensated by magnifying the panoramic imaging. Two indices, namely, cortical width at the gonion (GI) and below the mental foramen (MI), were measured bilaterally in all panoramic radiographs. All images were analyzed at a resolution of $300 \mathrm{dpi}$, and index values were calculated by applying linear measurements on the panoramic radiographs, with a tool provided by the software.

For GI measurements, one line was positioned parallel to the mandible ramus and another parallel to the mandible corpus. A third line starting from the intersection of the first two lines was finally drawn to obtain the measurement for the GI (Figure 1). For the MI measurements, a line was plotted perpendicular to other two lines drawn parallel to the inferior border of the mental foramen and to the inferior border of the mandible corpus (Figure 2).

Assessment of measurement reliability of replicate measurements of distances was carried out using the concordance correlation coefficient (CCC). One observer served as the main observer, and intraobserver reliability was estimated between measures performed 1 week apart.

2.3. Statistical Analyses. The Kruskal-Wallis test was applied to evaluate whether age groups data present statistical significance for each sex analyzed. Additionally, the MannWhitney test was applied to evaluate the significance level of differences between results for the same age groups of different sexes and to evaluate which age groups differ from one another with statistical significance. Pearson's correlation test was also performed in order to assess agreement between measurements for left and right sides.

A $5 \%$ level of significance was used to perform the statistical analysis.

\section{Results}

The data obtained for all 1287 panoramic radiographs analyzed are presented in Tables 1 and 2. Patients from age group 1 showed the highest values for both indices, and group 5 presented the lowest values for both indices. Intraobserver reproducibility was showed by the main observer, and the CCC ranged between 0.79 and 0.90 for the measurements.

Statistical analyses showed a significant difference between the results of each group analyzed in both male and female patients, using the Kruskal-Wallis test. A comparison between the results for groups 1 and 2 of different sexes also presented significant differences according to the MannWhitney test $(P<0.05)$. Correlation between the right and left sides was high for all variables $(r=0.85 ; P<0.001)$ with no significant differences demonstrated $(P>0.05)$. Therefore, the mean of the right and left sides was used in all statistical analyses.

An analysis of groups 3, 4, and 5 of female patients showed lower results for both, radiomorphometric indices in comparison with these same groups of male patients, with exception of MI values for group 4. Nevertheless, the difference found between these data showed no significance using the Mann-Whitney test $(P>0.05)$.

Mann-Whitney test was also applied to evaluate which age groups results differ from one another with statistical significance in male (Table 3 ) and female patients (Table 4). For GI, there were significant differences only between groups 2 and 3, and 3 and 4 in male patients, whereas for MI, significance was observed between groups 2 and 3, 3 and 4 , and 4 and 5 . In female patients results, for GI, there were significant differences between groups 1 and 2, 2 and 3, and 3 and 4, whereas for MI, significance was observed only between groups 3 and 4 and 4 and 5 .

\section{Discussion}

Several articles have described the importance of measuring radiomorphometric indices and BMD values for the prevention and diagnosis of osteoporosis [4-10]. Factors such as sex and age are established as determinants of individual bone quality. Smaller values of indices such as GI and MI in females were already expected owing to the bone loss of women after the fourth decade of life, a finding consistent with other authors $[7,8]$. The present results confirm significantly that a patient's age-related changes interfere with bone quality importantly in male patients as well supported by another similar study [9].

The comparison made by the present study among all radiomorphometric index results for all different patient age groups supports other articles in the literature $[10,11]$, insofar as younger men patients (group 1) showed significantly higher bone index measures for the indices studied, and among the female patients, group 5 showed the lowest index measures. Statistical analysis performed with MannWhitney test indicated a significant difference between some of the age groups results but in a different way for each index and patient sex. Interestingly, significant differences 


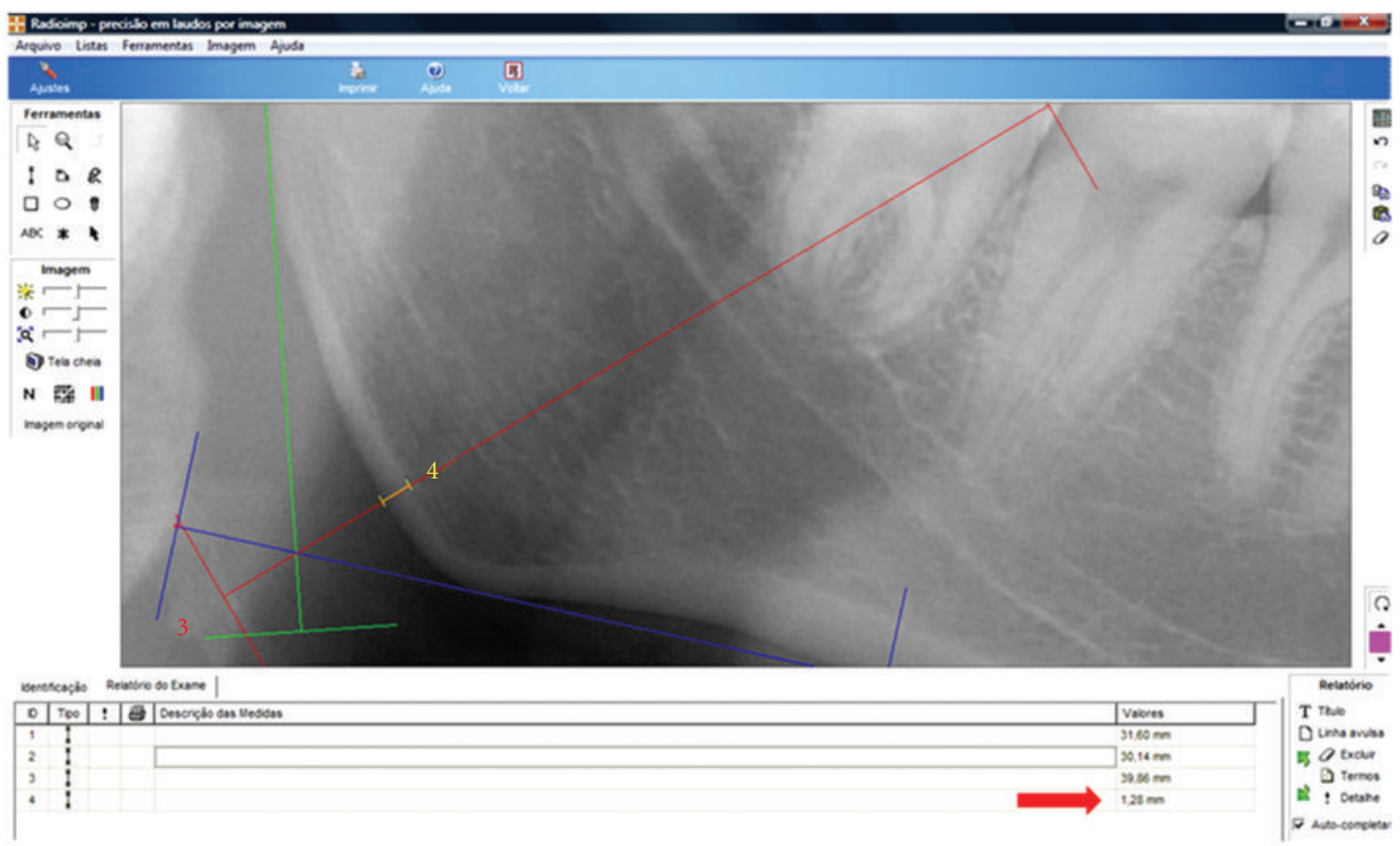

FIGURE 1: Measurement of the cortical thickness at the gonial region (gonial index-GI) using the software Radioimp.

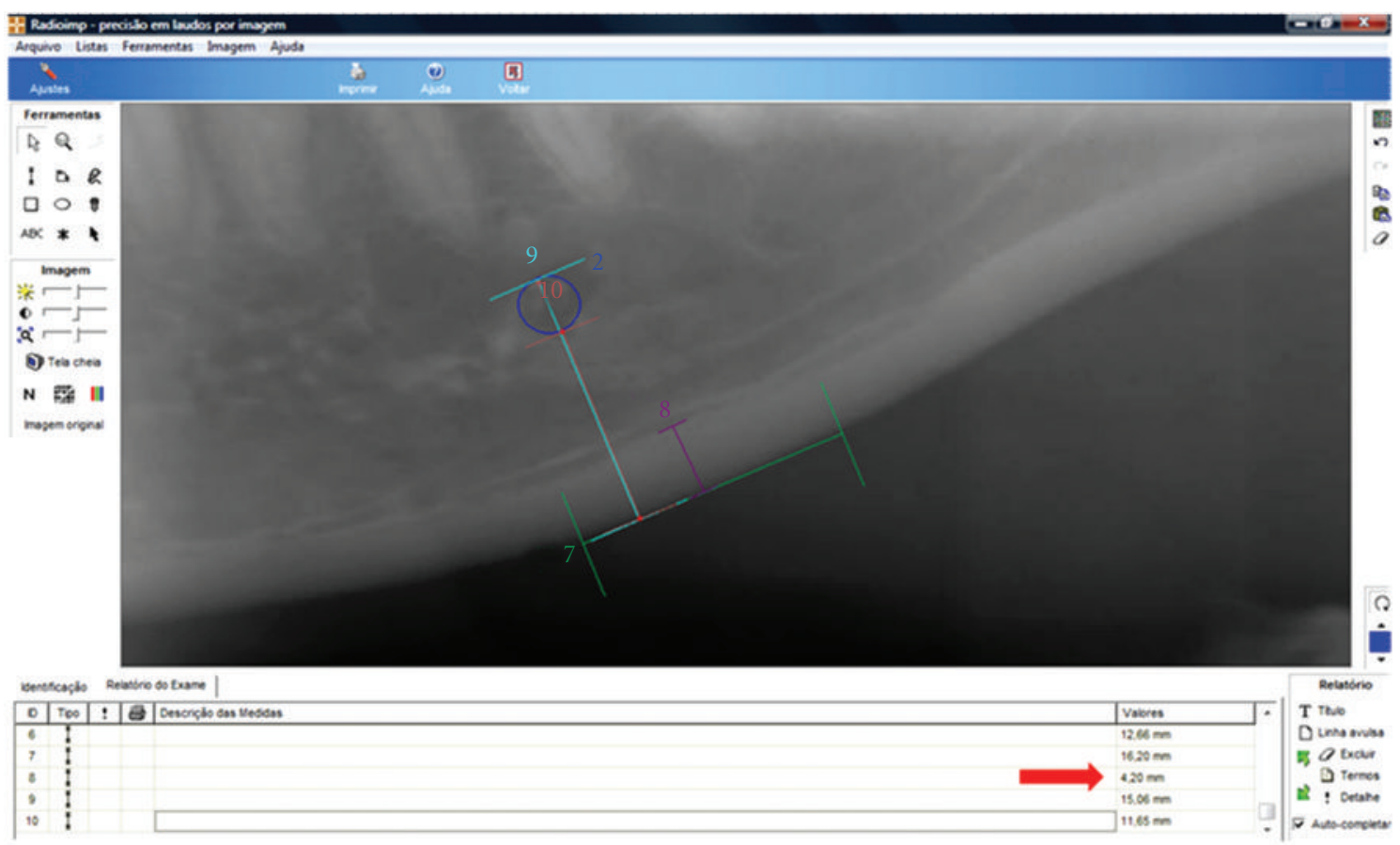

FIGURE 2: Measurement of the cortical thickness at the mental foramen (mental index-MI) using the software Radioimp.

were observed more often for MI in male patients and for GI in female patients, supporting that sex and age are factors that may influence BMD and the prognosis of osteoporosis, as described by other studies $[11,12]$. On the other hand, another study on panoramic radiomorphometric indices encountered no relation between patients' sex and the results for measurements in a Turkish population [10], which indicates that there still is a controversy in the literature. 
TABLE 1: Measurement results for male panoramic radiographs analyzed.

\begin{tabular}{|c|c|c|c|c|c|}
\hline Patient age groups & $\begin{array}{l}\text { Number of } \\
\text { cases }\end{array}$ & Mean age & $\begin{array}{l}\text { Means and standard } \\
\text { deviations for GI values }\end{array}$ & $\begin{array}{c}\text { Means and standard } \\
\text { deviations for MI values }\end{array}$ & $\begin{array}{c}\text { Kruskal-Wallis } \\
\text { test result }\end{array}$ \\
\hline 1 & $63(15.36 \%)$ & 18.79 & $1.37 \pm 0.37$ & $4.19 \pm 1.02$ & \multirow{5}{*}{$P<0.001$} \\
\hline 2 & $131(31.95 \%)$ & 31.38 & $1.33 \pm 0.16$ & $3.96 \pm 0.94$ & \\
\hline 3 & $147(35.85 \%)$ & 47.62 & $1.19 \pm 0.14$ & $3.55 \pm 0.54$ & \\
\hline 4 & $108(26.34 \%)$ & 64.92 & $1.11 \pm 0.16$ & $3.20 \pm 0.68$ & \\
\hline 5 & $54(13.17 \%)$ & 73.07 & $1.08 \pm 0.15$ & $3.07 \pm 0.64$ & \\
\hline
\end{tabular}

TABLE 2: Measurement results for female panoramic radiographs analyzed.

\begin{tabular}{lcccc}
\hline Patient age groups & $\begin{array}{c}\text { Number of } \\
\text { cases }\end{array}$ & Mean age & $\begin{array}{c}\text { Means and standard } \\
\text { deviations for GI values }\end{array}$ & $\begin{array}{c}\text { Means and standard } \\
\text { deviations for MI values }\end{array}$ \\
\hline 1 & $104(8.0 \%)$ & 19.16 & $1.25 \pm 0.23$ & $3.60 \pm 0.76$ \\
2 & $187(14.5 \%)$ & 27.54 & $1.20 \pm 0.24$ & $3.57 \pm 0.57$ \\
3 & $322(25.0 \%)$ & 49.27 & $1.14 \pm 0.25$ & $3.49 \pm 0.77$ \\
4 & $123(9.5 \%)$ & 66.24 & $1.08 \pm 0.23$ & $3.29 \pm 0.71$ \\
5 & $48(3.7 \%)$ & 75.06 & $1.06 \pm 0.26$ & $2.82 \pm 0.65$ \\
\hline
\end{tabular}

TABLE 3: Results for the statistical analysis of differences between male age groups results.

\begin{tabular}{ccc}
\hline Radiomorphometric index & $\begin{array}{c}\text { Age groups } \\
\text { compared }\end{array}$ & $\begin{array}{c}\text { Mann-Whitney } \\
\text { test result }\end{array}$ \\
\hline \multirow{3}{*}{ GI } & 1 and 2 & $P=0.1764$ \\
& 2 and 3 & $P<0.001$ \\
& 3 and 4 & $P<0.001$ \\
4 and 5 & $P=0.1024$ \\
\hline \multirow{3}{*}{ MI } & 1 and 2 & $P=0.1032$ \\
& 2 and 3 & $P<0.001$ \\
& 3 and 4 & $P<0.001$ \\
& 4 and 5 & $P=0.0095$ \\
\hline
\end{tabular}

TABLE 4: Results for the statistical analysis of differences between female age groups results.

\begin{tabular}{ccc}
\hline Radiomorphometric index & $\begin{array}{c}\text { Age groups } \\
\text { compared }\end{array}$ & $\begin{array}{c}\text { Mann-Whitney } \\
\text { test result }\end{array}$ \\
\hline \multirow{3}{*}{ GI } & 1 and 2 & $P=0.0036$ \\
& 2 and 3 & $P=0.0167$ \\
& 3 and 4 & $P=0.0058$ \\
& 4 and 5 & $P=0.2643$ \\
\hline \multirow{3}{*}{ MI } & 1 and 2 & $P=0.4704$ \\
& 2 and 3 & $P=0.1362$ \\
& 3 and 4 & $P=0.0037$ \\
& 4 and 5 & $P<0.001$ \\
\hline
\end{tabular}

This study also strove to make comparisons between results for each group of different sexes analyzed, considering that female patients showed lower results for MI and GI in all age groups, with the exception of MI values for group 4. However, statistical analysis of both radiomorphometric indices indicated a significant difference only between results for the males and females of groups 1 and 2. On the other hand, the results were closer for older male and female patients from groups 3, 4, and 5. Furthermore, the fact that male patients of age group 4 presented a lower mean value for $\mathrm{MI}$, when compared to the same group 4 of female patients, is an unexpected finding about the age-related changes of different sexes, since postmenopausal women are affected by bone loss and usually present lower values for panoramic radiomorphometric indices than other sex and age groups $[7,8]$. Therefore, this finding also stresses the importance of conducting further studies on osteoporosis in men.

In this study, no differences regarding dental status or other such data were assessed. This issue has been cited as a confusing parameter and not influential on MI values [12, 15]. Also, the thickness of the cortex at the gonial region has been found to present no marked differences in male patients between dentate and partially dentate and edentulous jaws [16].

The role of panoramic radiographs in the diagnosis of osteoporosis has already been described in the literature [12-16]. Some articles have suggested DXA as the gold standard method to evaluate bone quality and occurrence of osteoporosis $[11,14]$. However, findings of studies in the literature on both of these methods indicate that the use of panoramic radiography for predicting bone mineral density of the mandible also makes it possible to determine general skeletal bone mineral density loss in patients $[14,15]$.

Additionally, specific measurements such as that of the thickness of the mandibular inferior cortex in panoramic radiographs have also been suggested as a way of predicting low BMD in patients [15]. These radiomorphometric data, together with other criteria, such as clinical symptoms and/or family history of osteoporosis, could prove to be a promising instrument in assessing the risk of skeletal osteoporosis. 


\section{Conclusion}

The present paper supports the importance of age-related changes in mandibular radiomorphometric indices in identifying skeletal osteopenia and the role of osteoporosis in radiomorphometric index alterations, which can occur significantly and differently in male and female patients.

\section{References}

[1] S. C. White and D. J. Rudolph, "Alterations of the trabecular pattern of the jaws in patients with osteoporosis," Oral Surgery, Oral Medicine, Oral Pathology, Oral Radiology, and Endodontics, vol. 88, no. 5, pp. 628-635, 1999.

[2] C. F. Hildebolt, "Osteoporosis and oral bone loss," Dentomaxillofacial Radiology, vol. 26, no. 1, pp. 3-15, 1997.

[3] L. G. Raisz, "Physiology and pathophysiology of bone remodeling," Clinical Chemistry, vol. 45, no. 8, pp. 1353-1358, 1999.

[4] H. Devlin and K. Horner, "Mandibular radiomorphometric indices in the diagnosis of reduced skeletal bone mineral density," Osteoporosis International, vol. 13, no. 5, pp. 373-378, 2002.

[5] T. F. Tözüm and A. Taguchi, "Role of dental panoramic radiographs in assessment of future dental conditions in patients with osteoporosis and periodontitis," The New York State Dental Journal, vol. 70, no. 1, pp. 32-35, 2004.

[6] K. Horner, H. Devlin, C. W. Alsop, I. M. Hodgkinson, and J. E. Adams, "Mandibular bone mineral density as a predictor of skeletal osteoporosis," British Journal of Radiology, vol. 69, no. 827, pp. 1019-1025, 1996.

[7] A. Taguchi, M. Tsuda, M. Ohtsuka et al., "Use of dental panoramic radiographs in identifying younger postmenopausal women with osteoporosis," Osteoporosis International, vol. 17, no. 3, pp. 387-394, 2006.

[8] E. Klemetti, S. Kolmakov, P. Heiskanen, P. Vainio, and V. Lassila, "Panoramic mandibular index and bone mineral densities in postmenopausal women," Oral Surgery, Oral Medicine, Oral Pathology, Oral Radiology and Endodontology, vol. 75, no. 6, pp. 774-779, 1993.

[9] S. Dagistan and O. M. Bilge, "Comparison of antegonial index, mental index, panoramic mandibular index and mandibular cortical index values in the panoramic radiographs of normal males and male patients with osteoporosis," Dentomaxillofacial Radiology, vol. 39, no. 5, pp. 290-294, 2010.

[10] A. Gulsahi, B. Yüzügüllü, P. Imirzalioğlu, and Y. Genç, "Assessment of panoramic radiomorphometric indices in Turkish patients of different age groups, gender and dental status," Dentomaxillofacial Radiology, vol. 37, no. 5, pp. 288292, 2008.

[11] D. L. Broussard and J. H. Magnus, "Risk assessment and screening for low bone mineral density in a multi-ethnic population of women and men: does one approach fit all?" Osteoporosis International, vol. 15, no. 5, pp. 349-360, 2004.

[12] V. Dutra, J. Yang, H. Devlin, and C. Susin, "Radiomorphometric indices and their relation to gender, age, and dental status," Oral Surgery, Oral Medicine, Oral Pathology, Oral Radiology and Endodontology, vol. 99, no. 4, pp. 479-484, 2005.

[13] S. Ay, U. K. Gursoy, T. Erselcan, and I. Marakoglu, "Assessment of mandibular bone mineral density in patients with type 2 diabetes mellitus," Dentomaxillofacial Radiology, vol. 34, no. 6, pp. 327-331, 2005.

[14] E. Jagelaviciene, R. Kubilius, and A. Krasauskiene, "The relationship between panoramic radiomorphometric indices of the mandible and calcaneus bone mineral density," Medicina, vol. 46, no. 2, pp. 95-103, 2010.

[15] D. Ledgerton, K. Homer, H. Devlin, and H. Worthington, "Radiomorphometric indices of the mandible in a British female population," Dentomaxillofacial Radiology, vol. 28, no. 3, pp. 173-181, 1999.

[16] J. Bras, C. P. Van Ooij, and L. Abraham-Inpijn, "Radiographic interpretation of the mandibular angular cortex: a diagnostic tool in metabolic bone loss. Part I. Normal state," Oral Surgery, Oral Medicine, Oral Pathology, Oral Radiology and Endodontology, vol. 53, no. 5, pp. 541-545, 1982. 


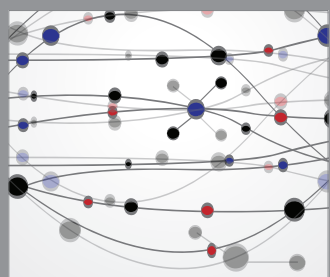

The Scientific World Journal
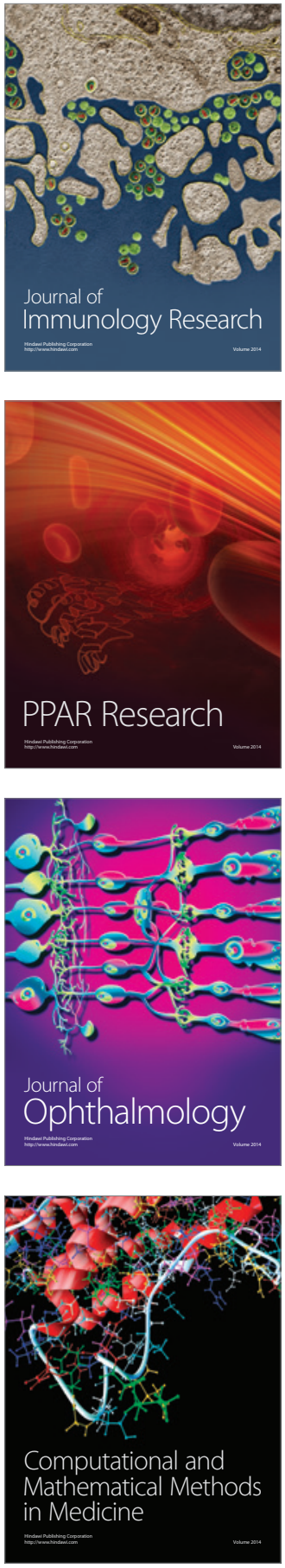

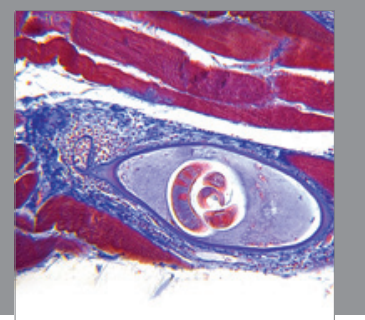

Gastroenterology

Research and Practice
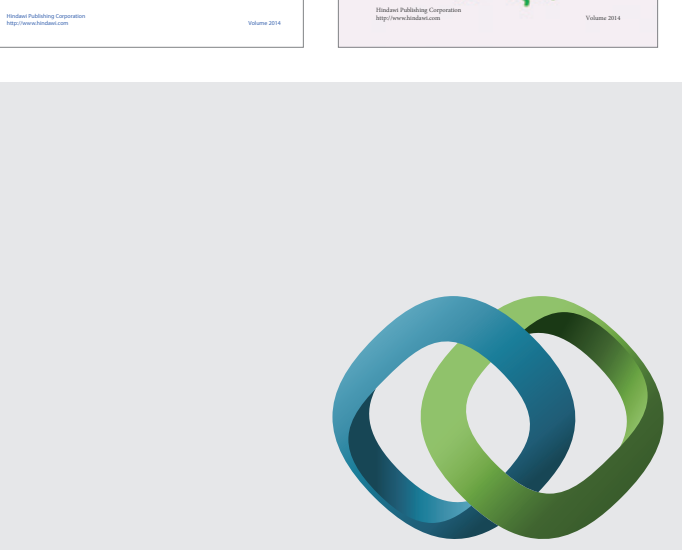

\section{Hindawi}

Submit your manuscripts at

http://www.hindawi.com
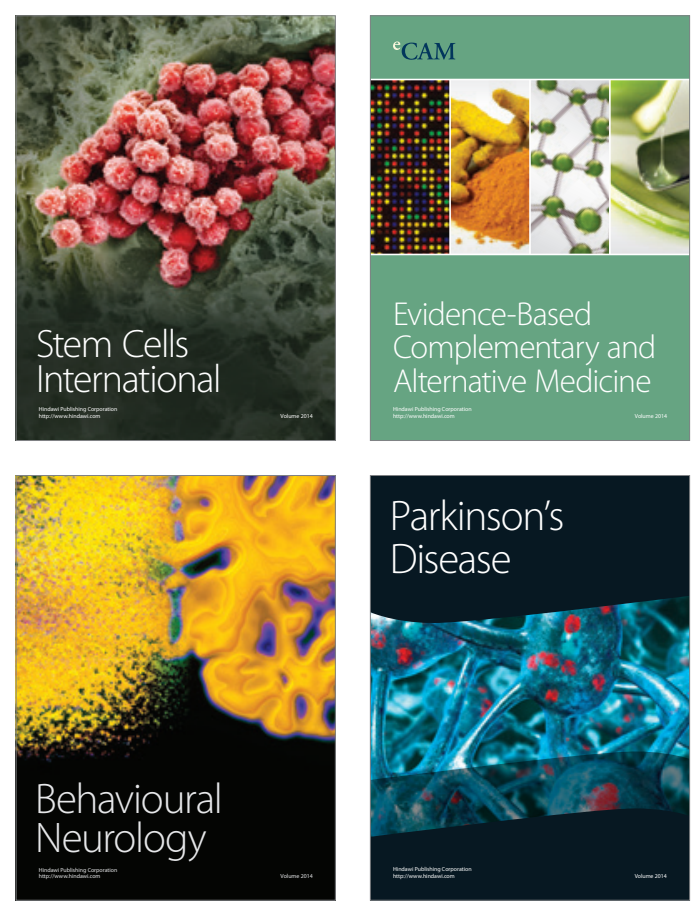

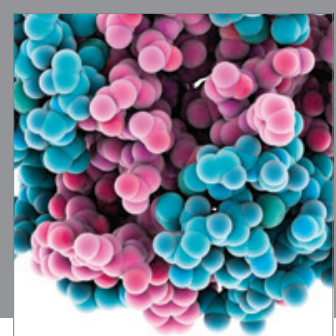

Journal of
Diabetes Research

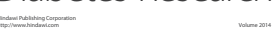

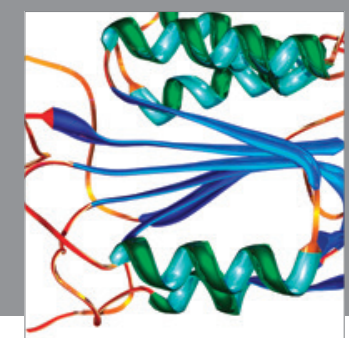

Disease Markers
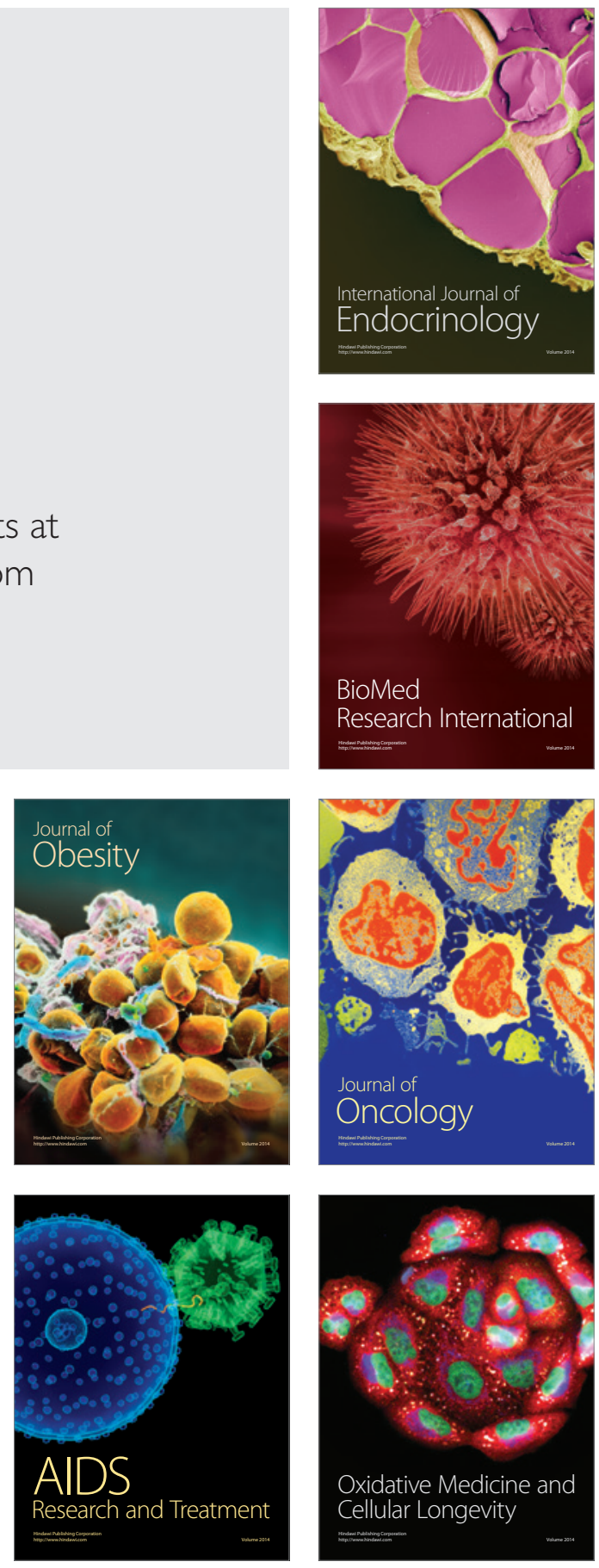